\title{
scGAD: single-cell gene associating domain scores for exploratory analysis of scHi-C data
}

\author{
Siqi Shen ${ }^{1}$, Ye Zheng ${ }^{2, *}$ and Sündüz Keleş ${ }^{1,3, *}$ \\ ${ }^{1}$ Department of Biostatistics and Medical Informatics, University of Wisconsin - Madison, Madison, WI 53706, USA, ${ }^{2}$ Biostatistics, \\ Bioinformatics and Epidemiology Program, Vaccine and Infectious Disease Division, Fred Hutchinson Cancer Research Center, Seattle, \\ WA 98109, USA, ${ }^{3}$ Department of Statistics, University of Wisconsin - Madison, Madison, WI 53706, USA. * Corresponding authors.
}

\begin{abstract}
Summary: Quantitative tools are needed to leverage the unprecedented resolution of single-cell high-throughput chromatin conformation (scHi-C) data and to integrate it with other single-cell data modalities. We present single-cell gene associating domain (ScGAD) scores as a dimension reduction and exploratory analysis tool for scHi-C data. ScGAD enables summarization at the gene level while accounting for inherent gene-level genomic biases. Low-dimensional projections with ScGAD capture clustering of cells based on their 3D structures. SCGAD enables identifying genes with significant chromatin interactions within and between cell types. We further show that scGAD facilitates the integration of scHi-C data with other single-cell data modalities by enabling its projection onto reference low-dimensional embeddings.

Availability: scGAD is part of the BandNorm R package at https://sshen82.github.io/BandNorm/articles/scGAD-tutorial.html. Contact: keles@stat.wisc.edu, yzheng23@fredhutch.org

Supplementary information: Supplementary data are available at Bioinformatics online.
\end{abstract}

\section{Introduction}

Single-cell technologies that profile chromatin conformation at the singlecell level emerged as promising approaches for high-resolution 3D genome characterization (Nagano et al., 2013; Stevens et al., 2017; Ramani et al., 2017; Li et al., 2019; Lee et al., 2019; Tan et al., 2021). Tools for specific scHi-C data inference tasks are gradually emerging (e.g., scHiCluster (Zhou et al., 2019), scHiC Topics (Kim et al., 2020), Higashi (Zhang et al., 2021), BandNorm and 3DVI (Zheng et al., 2021) for imputation and normalization of the sparse scHi-C data to advance de facto downstream analysis; SnapHiC (Yu et al., 2020) for chromatin loop detection; scHiCTools (Li et al., 2021) for quantifying cell-cell similarities). However, computational tools for extracting salient features of scHi-C data for integration with other single-cell data modalities, such as transcriptomics and epigenomics, are lacking. Here, we generalized the concept of gene-body associating domain (GAD) for bulk Hi-C data (Zhang et al., 2020) and developed the single-cell Gene Associating Domain (scGAD) scores and an accompanying R package. scGAD scores adjust for inherent genomic biases and summarize scHi-C data at the gene level. As a result, scGAD scores facilitate integrating $3 \mathrm{D}$ genome and other genomic data from single-cell technologies.

\section{Materials and methods}

We utilized scHi-C and scRNA-seq data of developing mouse cortex and hippocampus (Dip-C and MALBAC-DT data of Tan et al. (2021), respectively) and Paired-Tag data of adult mouse brain (Zhu et al., 2021) in our illustrations of scGAD. We first considered four variations of scGAD scores, namely $s c G A D_{\text {raw }}, s c G A D_{\text {local }}, s c G A D_{\text {regression, }}$, and $s c G A D_{\text {global }}$, to investigate whether they can accommodate inherent gene-level genomic biases (Supplementary Fig. 1 and Supplementary Note). Here, $s c G A D_{\text {raw }}$ is defined as the raw total number of interactions $R_{i j}$ for gene $i$ in cell $j$ without further normalization to illustrate the intrinsic genomic biases affecting the downstream analysis. As expected, $s c G A D_{\text {raw }}$ exhibits sequencing depth and gene length biases, where long genes and deeply sequenced cells dominate the cell clustering patterns (Supplementary Figs. 2-3). scGA $D_{\text {local }}$ leverages target gene's upstream and downstream regions of the same length as the target gene to estimate a gene-specific background. While this aims to adjust for the local background of the target gene, the neighboring regions might exhibit extreme sparsity, leading to zero number of interactions, or might harbor gene clusters, leading to overestimation of the background (Supplementary Figs. 2B-C). $s c G A D_{\text {regression }}$ first calibrates the sequencing depths of the cells and utilizes a Generalized Additive Model (GAM) to adjust for well known genomic biases, i.e., gene length, mappability, and GC content, with non-parametric smooth functions $s_{1}, s_{2}, s_{3}$. Specifically, we have $\widetilde{R}_{i j}=\frac{R_{i j}}{\sum_{i=1}^{M} R_{i j}}$,

$\widetilde{R}_{i j}=a+s_{1}\left(G C_{i}\right)+s_{2}\left(\right.$ Mappability $\left._{i}\right)+s_{3}\left(\right.$ GeneLength $\left._{i}\right)+\epsilon_{i j}$,

and $s c G A D_{\text {regression }}$ is the residual from this model. In contrast, $s c G A D_{\text {global }}$ removes potential gene-level biases implicitly by a standardization approach (Fig. 1A):

$$
s c G A D_{\text {global }}=\frac{\widetilde{R}_{i j}-\text { mean }_{i}}{\sqrt{\frac{1}{N-1} \sum_{j=1}^{N}\left(\widetilde{R}_{i j}-\text { mean }_{i}\right)^{2}}},
$$

where mean $_{i}=\frac{1}{N} \sum_{j=1}^{N} \widetilde{R}_{i j}$. Note that gene-wise standardization of the residuals from the GAM model is operationally equivalent to standardization of $\widetilde{R}_{i j}$, namely $s c G A D_{\text {global }}$. Hence, we kept $s c G A D_{\text {regression }}$ as unstandardized. We evaluated these variations for their performance in cell clustering and revealing relationships between the cell types. These comparisons revealed that $s c G A D_{\text {global }}$ performs the best by separating the cell types (Fig. 1B), almost as good as the full Hi-C contact matrices (Zheng et al., 2021), and recovers the known celltype relationships (Supplementary Figs. 4-5). We set $s c G A D_{\text {global }}$ as the formal definition of the scGAD scores for all the downstream analyses. 
bioRxiv preprint doi: https://doi.org/10.1101/2021.10.22.465520; this version posted October 24, 2021. The copyright holder for this preprint (which was not certified by peer review) is the author/funder, who has granted bioRxiv a license to display the preprint in perpetuity. It is made 2 available under aCC-BY-NC-ND 4.0 International license.

A
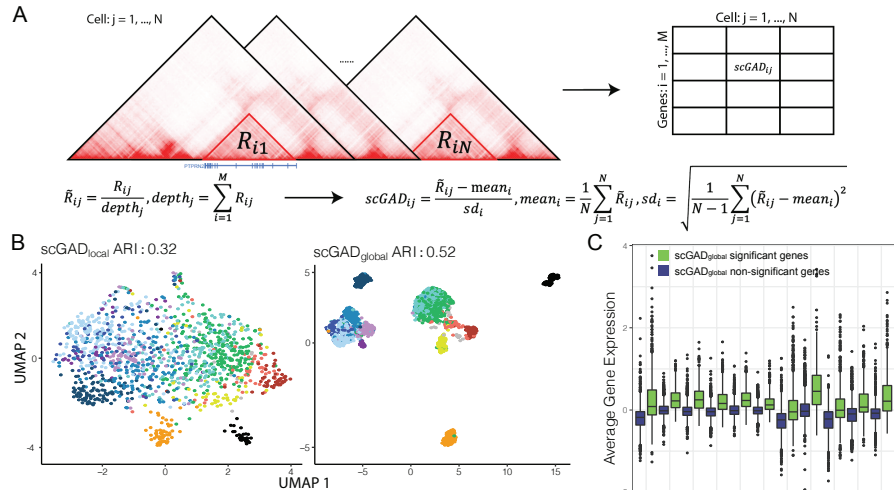

D
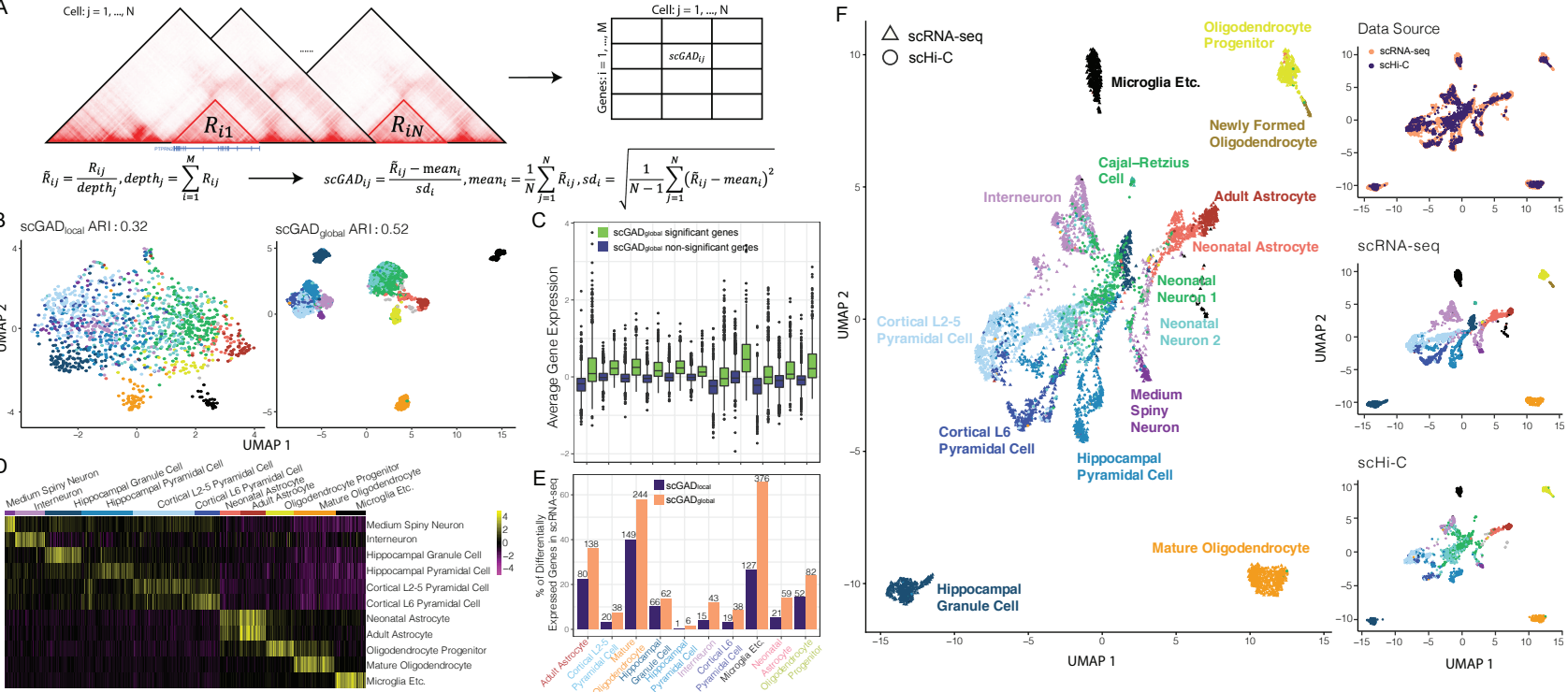

$\mathrm{scHi}-\mathrm{C}$

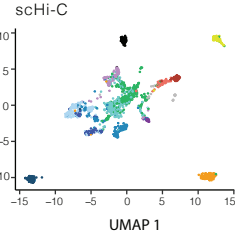

Fig. 1. A. Overview of scGAD score calculation. $R_{i j}$ is the total number of interactions within the promoter and body regions of gene $i$ in cell $j$. This calculation corresponds to best performing $s c G A D_{\text {global }}$ and is implemented as default in the R package. B. Comparison of the low-dimensional embeddings depicting cell-type separation by different scGAD score variations. Visualizations for all the variants are available in Supplementary Fig. 4. Adjusted Rank Index (ARI) quantifies the consistency between the $k$-means clustering of the cells in the low-dimensional embeddings (with top 50 principal components) and the true cell-type labels. C. Comparison of the gene expression between significant and non-significant marker genes detected by scGAD scores across cell types shared between scHi-C and scRNA-seq data. Each gene's scGAD score and expression are averaged over cells within each cell type. Genes with significantly high scGAD scores have significantly high gene expression (p-values from t-tests for each cell type $<10^{-26}$; Supplementary Fig. 7C). D. Average scGAD scores across cell-type-specific marker genes defined from scRNA-seq. Rows depict cell types in scRNA-seq, columns are the cells from the scHi-C data grouped by their cell types, and heatmap entries represent the average scores of scGAD marker genes. E. The percentage (y-axis) and the number (counts at the top of bars) of cell-type-specific marker genes defined from scRNA-seq and recovered by scGAD cell-type-specific marker gene detection. F. Projection of scHi-C data onto reference low-dimensional embeddings of scRNA-seq data with matching cell type labels.

\section{Result}

\section{scGAD: genes with abundant interactions}

Recent studies revealed that GAD formation is a chromatin feature of highly expressed genes (Zhang et al., 2020). We evaluated whether this feature is salient in the single-cell data. The correlations between the scGAD scores and expressions of genes were markedly higher, especially for the scRNA-seq marker genes, when both quantities were quantified in the same cell type compared to those in different cell types (p-value $<10^{-26}$, Supplementary Fig. 6). Next, we developed a permutation strategy to detect genes with significantly high scGAD scores as a means to infer highly expressed genes in a given cell type (Supplementary Note). When we evaluated genes with significantly high scGAD scores with the corresponding scRNA-seq data, they showed significantly higher expression levels compared to the genes with insignificant scGAD scores (Fig. 1C and Supplementary Fig. 7), illustrating how genes with high scGAD scores are highly expressed.

\section{scGAD: marker genes of cell types}

We observed that cell-type-specific marker genes defined from scRNAseq data displayed elevated scGAD scores (Supplementary Fig. 8). Furthermore, the average scGAD scores of these marker genes revealed cell-type-specific patterns (Fig. 1D and Supplementary Fig. 9). Leveraging the same marker gene detection procedure as in scRNA-seq analysis, we identified marker genes from scHi-C data with scGAD scores. We found that a large proportion of scRNA-seq marker genes overlapped with the scGAD marker genes (Fig. 1E), and the top scGAD significant marker genes yielded cell-type-specific gene expression patterns (Supplementary Fig. 10).

\section{scGAD: projection onto reference low-dimensional embeddings}

Finally, we asked whether scGAD scores can be exploited to project cells from scHi-C data onto a given reference low-dimensional embedding (e.g., from scRNA-seq; Fig. 1F). Projection onto the scRNA-seq embedding from the same system (i.e., with the exact same cell types) revealed that the cells originating from the same cell type but quantified by different data modalities were tightly clustered. Next, taking advantage of the Paired-Tag data, which included a larger number of cell types than the scHi-C data, we observed that scGAD facilitated an accurate projection of cells onto this larger space (Supplementary Figs. 11-12). This acrossmodality projection enables fast cell-type annotation for $3 \mathrm{D}$ genomics data and promotes integrative analyses of $3 \mathrm{D}$ genome structure, epigenomics, and transcriptomics to decipher gene regulation mechanisms at single-cell resolution.

In summary, scGAD provides a set of analysis tools to address the pressing needs for integrating scHi-C data with other single-cell data modalities.

\section{Funding}

This work was supported by NIH grants HG003747 and HG011371 to SK.

\section{References}

Kim, H.-J. et al. (2020). Capturing cell type-specific chromatin compartment patterns by applying topic modeling to single-cell hi-c data. PLoS computational biology, 16(9), e1008173.

Lee, D.-S. et al. (2019). Simultaneous profiling of 3D genome structure and DNA methylation in single human cells. Nature Methods, 16, 1-8. 
Li, G. et al. (2019). Joint profiling of DNA methylation and chromatin architecture in single cells. Nature Methods, 16.

Li, X. et al. (2021). schictools: A computational toolbox for analyzing single-cell hi-c data. PLoS computational biology, 17(5), e1008978.

Nagano, T. et al. (2013). Single-cell Hi-C reveals cell-to-cell variability in chromosome structure. Nature, $\mathbf{5 0 2}$.

Ramani, V.and Deng, X. et al. (2017). Massively multiplex single-cell Hi-C. Nature Methods, 14.

Stevens, T. et al. (2017). 3D structure of individual mammalian genomes studied by single cell Hi-C. Nature, 544, 59-64.

Tan, L. et al. (2021). Changes in genome architecture and transcriptional dynamics progress independently of sensory experience during postnatal brain development. Cell, 184(3), 741-758.

Yu, M. et al. (2020). Snaphic: a computational pipeline to map chromatin contacts from single cell hi-c data. https://www.biorxiv.org/content/10.1101/2020.12.13.422543v1.

Zhang, C. et al. (2020). taghi-c reveals $3 \mathrm{~d}$ chromatin architecture dynamics during mouse hematopoiesis. Cell Reports, 32(13), 108206.

Zhang, R. et al. (2021). Multiscale and integrative single-cell hi-c analysis with higashi. Nature Biotechnology.

Zheng, Y. et al. (2021). Normalization and denoising of single-cell hi-c data with bandnorm and $3 \mathrm{dvi}$. https://www.biorxiv.org/content/10.1101/2021.03.10.434870v1.

Zhou, J. et al. (2019). Robust single-cell hi-c clustering by convolution-and random-walk-based imputation. Proceedings of the National Academy of Sciences, 116(28), 14011-14018.

Zhu, C. et al. (2021). Joint profiling of histone modifications and transcriptome in single cells from mouse brain. Nature Methods, 18(3). 